\section{Renal Infarction Due to Spontaneous Renal Artery Dissection in Ehlers-Danlos Syndrome Type IV}

\section{To the Editor:}

Ehlers-Danlos syndrome (EDS) type IV is an autosomal dominant disorder caused by mutations in the gene for type III procollagen (COL3A1). The phenotype is characterized by thin translucent skin with visible veins, spontaneous rupture of arteries, uterus or intestines, characteristic facial features, and easy bruising ${ }^{1}$. Median survival is 48 years, with spontaneous arterial rupture the commonest cause of death ${ }^{2}$. We describe a case of renal infarction due to spontaneous renal artery dissection in EDS, highlighting the clinical presentation and management difficulties.

A 25-year-old man presented to an outlying facility in our hospital network with a 6-h history of acute severe right flank pain associated with 2 episodes of vomiting. Medical history was notable for a diagnosis of EDS type IV, diagnosed by dermal fibroblast culture at the age of 20 years. He dislocated his left shoulder at the age of 21 years and had a pneumothorax at age 22 years. His mother and brother had also been diagnosed with EDS type IV. He was a nonsmoker and denied cocaine use. Examination revealed the characteristic facies of his condition: translucent skin with visible veins, multiple intradermal pressure-point bruising, and mild hypermobility. Abdominal examination revealed tenderness in the right iliac fossa without guarding or rebound tenderness. Laboratory tests showed an elevated white blood cell count at $13.1 \times 10^{9} / 1$, with neutrophilia $11.2 \times 10^{9} / 1$. C-reactive protein was $8.5 \mathrm{mg} / \mathrm{l}$. Hematology and biochemistry were otherwise normal. Autoantibody and thrombophilia screens were normal, as were chest radiograph, electrocardiogram, transthoracic echocardiogram, and urinalysis. Computed tomography of the abdomen demonstrated multiple areas of renal infarction in the right kidney, predominantly in the lower pole but also involving the upper pole. Cortical involvement was estimated at $30 \%$. Dimercaptosuccinic acid scan demonstrated minimal if any uptake in the lower pole of the right kidney, with faint uptake in the upper pole. Relative uptake was estimated at $23 \%$ for the right kidney and $77 \%$ for the left kidney. Magnetic resonance angiogram confirmed the renal infarctions (Figure 1A) and demonstrated right renal artery dissection (Figure 1B, Figure 2). Initial treatment consisted of analgesia, intravenous antibiotics, and unfractionated heparin. Following transfer to our center, anticoagulation was stopped. His pain decreased somewhat and he had no further vomiting. He was subsequently transferred back to his local hospital.
EDS is an inherited disorder of connective tissue caused by defective collagen production. Several subtypes are recognized today, including type IV. The friability of the vasculature in type IV EDS commonly results in arterial dissection or spontaneous arterial rupture. Infarction is a rare complication. Two previous cases of EDS associated with renal infarction have been reported. Yigal, et al, described a patient who presented with right thromboembolic renal infarctions due to a right renal artery aneurysm that was managed conservatively ${ }^{3}$. Gelbmann, et al described the case of a young woman who presented with renal infarction associated with spontaneous rupture of the liver and pneumothorax ${ }^{4}$. An additional case in the French language literature described a woman who had renal artery dissection in pregnancy ${ }^{5}$. The management of renal infarction is dependent on the etiology. Anticoagulation is indicated in cases resulting from cardioembolic sources ${ }^{6}$. Management of renal artery dissection resulting in renal infarction is controversial, with conservative management, anticoagulation, and surgical and endovascular revascularization, as described ${ }^{7}$. The use of both unfractionated and low molecular weight heparins is associated with an increased risk of hemorrhagic complications ${ }^{8,9}$. The use of anticoagulation in EDS type IV may increase the risk of fatal hemorrhage unrelated to the thrombotic presentation. In the absence of randomized controlled trial data in this rare complication of a rare disease, we advocate treatment decisions on a case by case basis. In cases of non-life-threatening thromboembolic disease, the risks may outweigh the benefits of anticoagulation in both the short and long terms.

Our case demonstrates a rare cause of renal infarction, which should be added to the differential diagnosis. To our knowledge this is the first report of a case of renal artery dissection presenting with renal infarction in EDS. The optimum management of renal infarction in Ehlers-Danlos syndrome type IV remains to be determined.

RICHARD CONWAY, MD, Rheumatology Specialist Registrar; DIANE BERGIN, MD, Consultant Radiologist; ROBERT J. COUGHLAN, MD, Consultant Rheumatologist; JOHN J. CAREY, MD, Consultant Rheumatologist, Departments of Rheumatology and Radiology, Galway University Hospitals, Merlin Park, Galway, Ireland. Address correspondence to Dr. R. Conway, Department of Rheumatology, Galway University Hospitals, Merlin Park, Galway, Ireland.

E-mail: drrichardconway@gmail.com

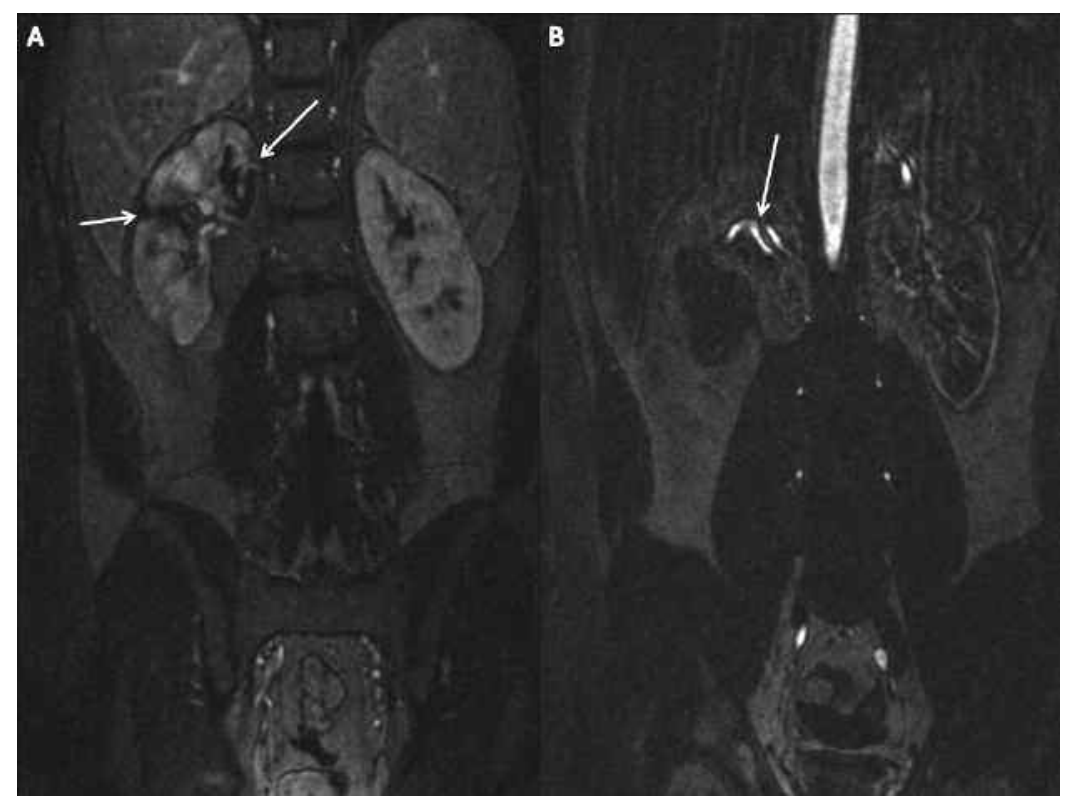

Figure 1. Coronal magnetic resonance renal angiogram demonstrating nonenhancing renal parenchymal infarcts (A, arrows) and right renal artery dissection (B, arrow). 


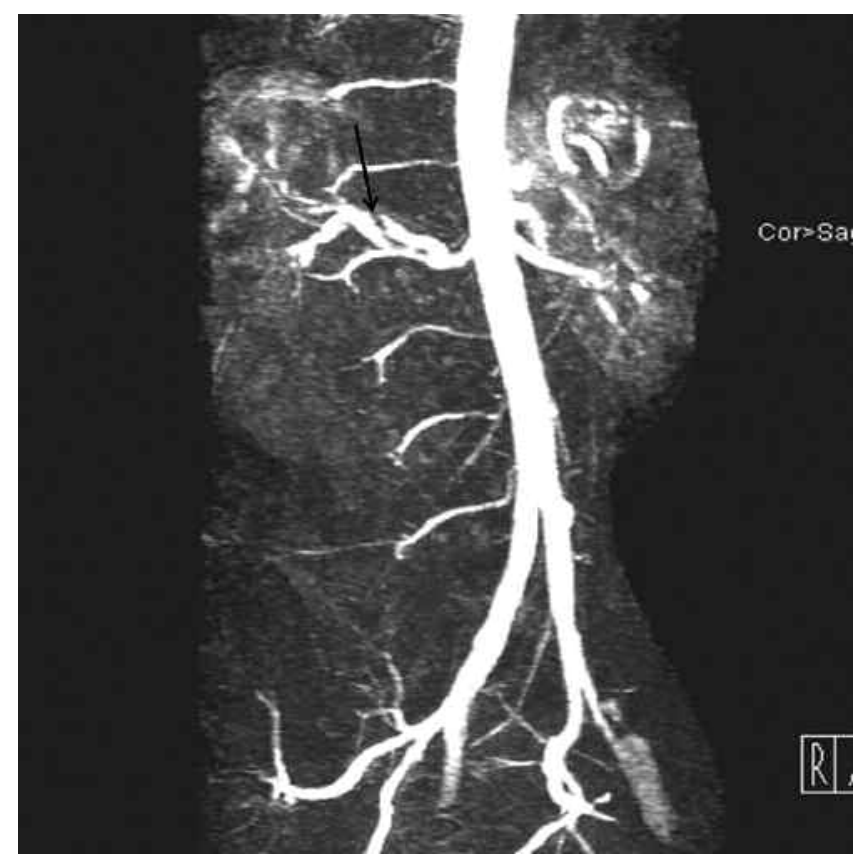

Figure 2. Magnetic resonance imaging abdominal aortic angiogram showing right renal artery dissection (arrow).

Dr. Conway reports receiving research funding from Roche Ireland Limited and UCB (Pharma) Ireland Ltd, and honoraria from Merck.

\section{REFERENCES}

1. Beighton P, De Paepe A, Steinmann B, Tsipouras P, Wenstrup RJ. Ehlers-Danlos syndromes: Revised nosology, Villefranche, 1997. Am J Med Genet 1998;77:31-7.
2. Pepin M, Schwarze U, Superti-Furga A, Byers PH. Clinical and genetic features of Ehlers-Danlos syndrome type IV, the vascular type. N Engl J Med 2000;342:673-80.

3. Yigal A, Tal H, Nurit H, Tova CS, Hershcovici T. Thromboembolic renal infarction due to a renal artery aneurysm in a patient with Ehlers-Danlos syndrome type IV. Eur J Intern Med 2006;17:377-9.

4. Gelbmann CM, Köllinger M, Gmeinwieser J, Leser HG, Holstege A, Schölmerich J. Spontaneous rupture of liver in a patient with Ehlers Danlos disease type IV. Dig Dis Sci 1997;42:1724-30.

5. Debie B, Hammer F, Dahan K, Feyaerts A, Jasienski S, Opsomer $\mathrm{RJ}$, et al. Rupture of the renal artery and renal parenchyma in a pregnant woman with vascular form of Ehlers-Danlos syndrome [French]. Prog Urol 2005;15:303-5.

6. Hazanov N, Somin M, Attali M, Beilinson N, Thaler M, Mouallem $\mathrm{M}$, et al. Acute renal embolism. Forty-four cases of renal infarction in patients with atrial fibrillation. Medicine 2004;83:292.

7. Ramamoorthy SL, Vasquez JC, Taft PM, McGinn RF, Hye RJ. Nonoperative management of acute spontaneous renal artery dissection. Ann Vasc Surg 2002;16:157-62.

8. Cohen M, Demers C, Gurfinkel EP, Turpie AG, Fromell GJ, Goodman S, et al. A comparison of low-molecular-weight heparin with unfractionated heparin for unstable coronary artery disease. Efficacy and Safety of Subcutaneous Enoxaparin in Non-Q-Wave Coronary Events Study Group. N Engl J Med 1997;337:447-52.

9. Levine M, Gent M, Hirsh J, Leclerc J, Anderson D, Weitz J, et al. A comparison of low-molecular-weight heparin administered primarily at home with unfractionated heparin administered in the hospital for proximal deep-vein thrombosis. N Engl J Med 1996;334:677-81.

J Rheumatol 2012;39:1; doi:10.3899/jrheum.111034 